\title{
Outcome of Palliative and Radical Radiotherapy in Patients with Oral Squamous Cell Carcinoma - a Retrospective Study
}

\author{
Narendra Prakash Rai ${ }^{1}$, Darshan Devang Divakar ${ }^{2 *}$, Abdulaziz Abdullah Al \\ Kheraif $^{2}$, Ravikumar Ramakrishnaiah ${ }^{2}$, Shabil Mohamed Mustafa ${ }^{3}$, Durgesh \\ BH $^{2}$, Santhosh Basavarajappa ${ }^{2}$, Aftab Ahmed Khan ${ }^{2}$
}

\begin{abstract}
Background: The treatment selection for the oral squamous cell carcinoma remains controversial. Radiation therapy or surgical excision of the lesion can be applied as the sole treatment or it can be used in combination with other treatment modalities. Radiotherapy is considered to be the safest of all the treatment modalities and can be used in several situations for oral and oropharyngeal cancers. The aim of this study was to evaluate the survival outcome differences in patients treated with radical and palliative radiotherapy as the primary treatment modality. Materials and Methods: The study included a total of 47 patients with oral cancer reporting to our hospital between years 2009 to 2010 . The age group for the selected patients was more than 65 years, treated with radical and palliative radiotherapy with no prior surgical interventions. Patients were evaluated till Dec 2013 for overall survival time. Results: Twenty nine patients were treated with radical radiotherapy as main stay of treatment, out of which 21 died during the follow up time with median survival of $352 \pm 281.7$ days with 8 patients alive. All the 16 patients were dead who received palliative radiotherapy with a median survival time of $112 \pm 144.0$ days. Conclusions: This retrospective study showed improved overall survival time, loco regional control rates and reduced morbidity in patients treated with radical radiotherapy when compared to patients treated with palliative radiotherapy.
\end{abstract}

Keywords: Long term survival - oral cancer - palliative radiotherapy - radical radiotherapy

Asian Pac J Cancer Prev, 16 (16), 6919-6922

\section{Introduction}

Head and neck squamous cell carcinoma is the most common malignancy with more propensity to occur in the oral cavity (Patel et al., 2014). Oral cancer is one of the major health hazards in India and it accounts for approximately $30-40 \%$ of all cancer cases and in these cases $80 \%$ of them are oral squamous cell carcinoma (Krishnan et al., 2014). Recently there has been a significant increase in the incidence of oral cancer which could be attributed to premature tobacco abuse (ICMR, 2001; Moore and Tsuda, 2002; Patel et al., 2014; Kumar and Muniyandi, 2015) in teenagers along with use of alcohol (Al-Attas et al., 2014), while in females most commonly it can be due to tobacco chewing and poor oral hygiene (95\%) (Moore and Tsuda, 2002). Carcinogen exposure and proliferative stimuli, are the two etiological factors responsible for the malignant transformation (Moore, 2009). Metastasis and unknown secondaries are well documented and widely recognized cause for mortality and morbidity in such patients.

While surgery has already carved a niche for itself in the field of cancer treatment, radiotherapy, although proven to be equally effective in certain cases still does not enjoy the benefit of widespread awareness. The advanced stages make the prognosis poorer for patients with oral squamous cell carcinoma despite of advancement in the different treatment modality and multimodal treatment regimens (Krishnan et al., 2014).

Different treatment facilities for cancer and modalities like radiation therapy, still are an enigma to many practicing doctors and thus the need arise not only to acquaint ourselves with the different treatment options but also to judge those options on the basis of their outcomes (Moore, 2013).

Greater tumor destruction is possible when total radiation doses are fractioned into multiple small doses. Radical radiotherapy intends to cure the disease whereas 


\begin{tabular}{|c|c|c|c|c|c|c|c|c|}
\hline \multirow[t]{3}{*}{ Mode of Radiotherapy } & \multicolumn{4}{|c|}{ Mean } & \multicolumn{4}{|c|}{ Median } \\
\hline & \multirow[t]{2}{*}{ Estimate } & \multirow[t]{2}{*}{ Std. Error } & \multicolumn{2}{|c|}{ 95\% Confidence Interval } & \multirow[t]{2}{*}{ Estimate } & \multirow[t]{2}{*}{ Std. Error } & \multicolumn{2}{|c|}{ 95\% Confidence Interval } \\
\hline & & & Lower Bound & Upper Bound & & & Lower Bound & Upper Bound \\
\hline $\mathrm{P}$ & 314.625 & 88.977 & 140.23 & 489.02 & 112 & 144 & 0 & 394.24 \\
\hline $\mathrm{R}$ & 672.584 & 113.16 & 450.79 & 894.378 & 352 & 281.656 & 0 & 904.046 \\
\hline Overall & 541.807 & 82.541 & 380.026 & 703.587 & 306 & 92.55 & 124.601 & 487.399 \\
\hline
\end{tabular}

Table 1. Mode of Radiotherapy

\begin{tabular}{clccc}
\hline & & Frequency & Percent $\%$ & Valid Percent \% \\
\hline Valid & $\mathrm{P}$ & 16 & 34 & 34 \\
& $\mathrm{R}$ & 31 & 66 & 66 \\
& Total & 47 & 100 & 100 \\
\hline
\end{tabular}

palliative radiotherapy is aimed at alleviating local symptoms and improving the quality of life (Nitin Pratap Shishodia et al., 2015). Decisions on the type of treatment administered to patient has to be decided by the oncologist panel before the start of any treatment.

The approach we have used in this study aims to evaluate the long-term survival of patients suffering from oral squamous cell carcinoma treated with palliative and radical radiotherapy.

\section{Materials and Methods}

Study included total 47 patients who reported to Malabar Cancer Centre and Hospital, Kerala, India between 2009 to 2010. All the patients were suffering from oral cancer which was histopathologically confirmed. Patient details, progression of the treatment, follow up and treatment outcomes of these patients were analyzed from the outpatient record section. All the patients selected were not less than 65 years of age and were treated with radical and palliative radiotherapy as main treatment modality, with no prior surgical interventions; patients were also on adjuvant chemotherapeutic drugs. Patients were evaluated till Dec 2013 for overall survival time. Exclusion criteria for the study was any previous history of surgical intervention for the treatment of oral cancer and age less than 65 years. Whereas, inclusion criteria was histologically confirmed oral squamous cell carcinoma with no prior surgical interventions.

\section{Results}

Descriptive data is shown in the form of frequency and percentage. The values of this study were subjected to statistical analysis using SPSS software IBM version 16. Statistical Analysis was done using Pearson Chi-Square and Fisher's Exact test.

Forty seven patients were included in the study who reported to hospital during year Jan 2009 to Dec 2010 and diagnosed as having oral squamous cell carcinoma. Out of those 16 patients received palliative radiotherapy as main treatment modality and 31 patients with radical radiotherapy Table 1 . Palliative radiotherapy was planned for these patients due to their poor general condition and
Table 3. Sex Distribution

\begin{tabular}{cccccc}
\hline & & Frequency & Percent $\%$ & $\begin{array}{c}\text { Valid } \\
\text { Percent } \%\end{array}$ & $\begin{array}{c}\text { Cumulative } \\
\text { Percent } \%\end{array}$ \\
\hline Valid & F & 18 & 38.3 & 38.3 & 38.3 \\
& M & 29 & 61.7 & 61.7 & 100 \\
& Total & 47 & 100 & 100 & \\
\hline
\end{tabular}

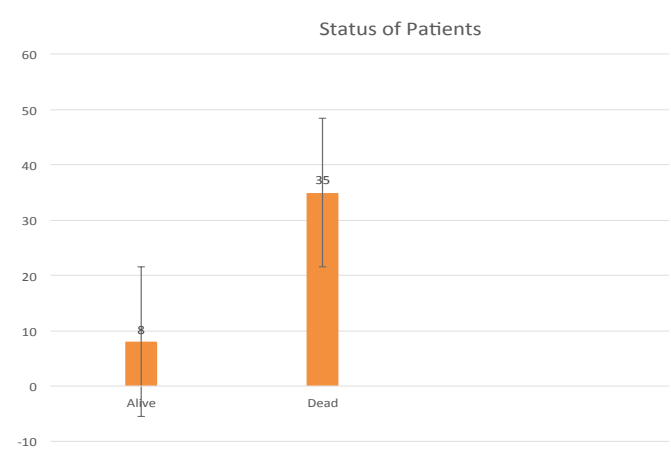

Figure 1. Status of Patients

bad prognosis and to alleviate the condition so that quality the of life of these patients can be improved. All the 16 patients died with median survival time of $112 \pm 144.0$ days during the follow up period, rest 29 patients were treated with radical radiotherapy with median survival time of $352 \pm 281.6$ days Table 2, out of which 21 patients died and 8 patients were leading healthy life with no signs of progressive or recurrence of the lesion, Figure 1 shows the status of patients. Data was not available for 2 patients. Out of total 47 patients 29 were male and 18 were female patients Table 3 .

\section{Discussion}

Radiation is the transfer of energy through space and matter. It can be used as curative treatment modality for head \& neck cancer patients or it can also be used in patients with poor operative conditions with extensive lesions, this will preserve patient's structural and functional integrity of tissues. Radiation therapy is a speciality in which ionizing radiation is used in the treatment of malignant diseases. High energy radiation is used for the therapeutic purposes.

Long term survival for patients with oral cancer is improving with advancement of various treatment modalities but their result remains suboptimal which is due to locally advanced disease at the time of presentation with vast majority of cases of stage III and IV (Abbasi et al., 2014; Krishnan et al., 2014)

In earlier days surgery was considered to be the prime 
Outcome of Palliative and Radical Radiotherapy in Patients with Oral Squamous Cell Carcinoma - A Retrospective Study

treatment modality for all head \& neck cancer treatment but that led to significant morbidity of patients. In the era of advancement, radiotherapy has replaced the surgical treatment modalities for the primary local head \& neck cancers, before it was only restricted to cases which were unresectable or cases with palliative treatments.

Chances of recurrences are more in patients treated alone with surgery so in those cases adjuvant radiotherapy or concurrent chemo-radiotherapy can also be considered for better loco-regional control of these disease.

For head and neck cancer patients, treatment decisions are based on the probability of cancer control, the expected treatment morbidities, the social factors, other comorbidities and physiological reserve of the patient. Early to intermediate stage cases can be managed either by radiation or surgery alone.

Radiation damages the DNA of cancer cells and destroys the ability of these cancer cells to reproduce and repopulate. A typical course of radiation therapy for head and neck cancer is delivered 5 days per week, over 7 weeks for 35 fractions.

Radiotherapy is indicated in early stage diseases, inoperable cases (medical contra-indication) and in cases where surgery is morbid.

Radiation beam can be fractioned in several doses and given to cure the disease, is known as radical radiotherapy, i.e 60-70 Gy for 35 fractions for 7 weeks and fractioned doses given just to alleviate the condition is called as palliative radiotherapy and is given as $8 \mathrm{~Gy}$ for 1 fraction or $20 \mathrm{~Gy}$ for 5 fractions, depending on tumor.

Multiple smaller fractions of radiation therapy control the rate of tumor growth better than single large doses. Coutard (Fonseca RJ, 2000) found that small superficial lesions can be controlled by single large fractions of radiation therapy whereas more deep seated tumors are best controlled by fractionation of the radiation beam. This method of fractionation helped radiotherapists to control many advanced cancer cases which were difficult to control by single large doses.

The various types of fractionation are:

1. Conventional fractionation-180-200 cGy/d, 5 fractions per week to a total dose of 6000-7000 Gy.

2. Hyper fractionation-110-125 cGy bid, $5 \mathrm{~d} / \mathrm{wk}$ to 7000-8000 cGy; no planned interruptions.

3. Continuous Accelerated fractionation-120-180 cGy bid or tid, $7 \mathrm{~d} /$ wk to $5000-5400 \mathrm{cGy}$; no planned interruptions.

4. Accelerated hyper fractionation-140-160 cGy bid, for $5 \mathrm{~d} / \mathrm{wk}$ to $6600-7200 \mathrm{cGy}$; 1-2 wk break in between treatment.

5. Concomitant-Boost technique-180-200 cGy/d for 5 $\mathrm{d} /$ wk to $3500-4500 \mathrm{cGy}$; followed by $150-180$ cGy twice daily "conedown" boost radiotherapy to 6600-7200 cGy with no interruptions in between.

6. Hypo fractionation-250-300 cGy/d, 3-4 d/wk to 5000-7000 cGy; no planned interruptions.

7. Split course therapy-200-300 cGy/d for $5 \mathrm{~d} / \mathrm{wk}$ to 6000-7200 cGy; 1-2 week break in between.

Surgical treatment and radiation therapy are equally effective for small and superficial tumors and can be used singly or in combination of both for the treatment of the patients. Other factors such as complications, cost, convenience and compliance, long term sequelae of treatment, site, location, and status of node should also be taken care before planning the treatment for such patients.

Both the treatment modalities surgical as well as radiation therapy are considered to be complementary rather than competitive to each other having its own applications and morbidities. Cancer surgery leads to removal of the diseased area resulting in structural and functional disability whereas radiation therapy leaves the anatomy intact but this could lead to destruction of its physiology and function.

This present study was conducted to evaluate the long term survival outcome for the patients treated with palliative and radical radiotherapy. Study shows that the median survival time period for the 29 patients treated with radical radiotherapy was of $352 \pm 281.7$ days with 8 patients alive. Whereas all the 16 patients were dead who received palliative radiotherapy with median survival time of $112 \pm 144.0$ days after 3 year follow up period.

Preoperative radiotherapy is mainly indicated in cases with involvement of node below first level of lymphatics, multiple positive nodes and nodes that persist 6-8 weeks after radiotherapy. Advantages of preoperative radiotherapy are inoperable lesions may be converted to operable, extent of surgery may be decreased and blood supply at the time of radiotherapy is intact.

(Ryan and Nancy, 2009) reported the results that randomized 295 patients with unresectable head and neck cancer with a median follow-up of 41 months, concurrent cisplatin and radiotherapy without a planned treatment break resulted in superior 3-year overall survival.

In another case series of 505 patients done by (Mohanti et al.,2004), short course palliative radiotherapy (20Gy in five fractions over five days) provided durable symptom relief in $55 \%$ of patients.

Fractionation schedules and newer treatment techniques are the result of understanding and betterment of radiation biology. In the advancement of treatment modalities, our goal should be focused to provide maximum ratio of therapeutic index between normal and tumor tissues.

In conclusion, the mission of any advancement in cancer treatment is to play a vital role in achieving world class cancer services, and to ensure better treatment outcomes. The goals of treatment should include maximum tumor control while maintaining structural and functional integrity of tissues and patients quality of life. Long duration studies with large sample size should be conducted with routine follow up to see the proper outcome of both the radiotherapy modalities.

\section{Acknowledgements}

The project was financially supported by King Saud University, Vice Deanship of Research Chairs Kingdom of Saudi Arabia, Riyadh.

We would like to thank Dr. Satheesan Balasubramanian, Director and Dr. Santam Chakaraborthy, Assistant Professor, Department of radiation oncology, Malabar Cancer Centre, Kerala, India for providing necessary facilities and guidance to carry out this study. 


\section{References}

Abbasi M M, Esfahlan RJ, Monfaredan A, et al (2014). Oral and IV dosages of doxorubicin-methotrexate loadednanoparticles inhibit progression of oral cancer by downregulation of matrix methaloproteinase 2 expression in Vivo. Asian Pac J Cancer Prev, 15, 10705-11.

Al-Attas S A, Ibrahim S S, Amer H A, Darwish Z E, Hassan M $\mathrm{H}$ (2014). Prevalence of potentially malignant oral mucosal lesions among tobacco users in jeddah, Saudi Arabia. Asian Pac J Cancer Prev, 15, 757-62.

Fonseca RJ (2000) Oral and Maxillofacial Surgery. Surgical Pathology 1st edition. Philadelphia, Pennsylvania; Saunders publication.

Indian Council of Medical Research (2001). ICMR Bulletin. National cancer registry of the indian council for medical research annual report, New Delhi, India.

Krishnan R, Thayalan DK, Padmanaban R, et al (2014). Association of serum and salivary tumor necrosis factor- $\alpha$ with histological grading in oral cancer and its role in differentiating premalignant and malignant oral disease. Asian Pac J Cancer Prev, 15, 7141-8.

Kumar S, Muniyandi M (2015). Tobacco Use and oral leukoplakia: cross-sectional study among the gond tribe in madhya pradesh. Asian Pac J Cancer Prev, 16, 1515-8.

Mohanti BK, Umapathy H, Bahadur S, Thakar A, Pathy S (2004). Short course palliative radiotherapy of $20 \mathrm{~Gy}$ in 5 fractions for advanced and incurable head and neck cancer: AIIMS study. Radiother Oncol, 71, 275-80.

Moore MA, Tsuda H (2002). Smoking-related research in the asian-pacific countries. Asian Pac J Cancer Prev, 3, 291-304.

Moore MA (2009). Diverse influences of dietary factors on cancer in asia. Asian Pac J Cancer Prev, 10, 981-6.

Moore MA (2013). Overview of cancer registration research in the Asian Pacific from 2008-2013. Asian Pac J Cancer Prev, 14, 4461-84.

Shishodia NP, Divakar DD, Al Kheraif AA, et al (2015). End stage palliative care of head and neck cancer: a case study. Asian Pac J Cancer Prev, 16, 1255-8.

Patel SS, Shah KA, Shah MJ, Kothari KC, Rawal RM (2014). Cancer stem cells and stemness markers in oral squamous cell carcinomas. Asian Pac J Cancer Prev, 15, 8549-8556.

Ryan J. Burri, Nancy Y. Lee (2009). Concurrent chemotherapy and radiotherapy for head and neck cancer. Expert Rev Anticancer Ther, 9, 293-302. 